\title{
Recommendation Optimization of Physical Education for Developing the Intelligence of Autistic Children following Intelligent Collaborative Filtering Algorithm
}

\author{
Hongzhong $\mathrm{Hao}^{1}$ and Sheng $\mathrm{Hu}$ iD $^{2}$ \\ ${ }^{1}$ School of Physical Education, Huaibei Normal University, Huaibei 235000, Anhui, China \\ ${ }^{2}$ School of Education, Huaibei Normal University, Huaibei 235000, Anhui, China \\ Correspondence should be addressed to Sheng Hu; hus@chnu.edu.cn
}

Received 6 December 2021; Revised 24 December 2021; Accepted 29 December 2021; Published 21 January 2022

Academic Editor: Ateeq Rehman

Copyright (C) 2022 Hongzhong Hao and Sheng Hu. This is an open access article distributed under the Creative Commons Attribution License, which permits unrestricted use, distribution, and reproduction in any medium, provided the original work is properly cited.

\begin{abstract}
Autism, a developmental disorder affecting social and communication skills, differs from most the mental handicap in showing a characteristic pattern of poor, intact, and even superior cognitive abilities. This study aims to solve the mismatch of the teaching content and mental health education for autistic children. Inspired by artificial intelligence, an improved neural network matrix factorization (NeuMF) model is designed based on the theory of collaborative filtering, and time data is added to improve the NeuMF by using the K-means clustering algorithm. Several evaluation indexes such as root mean square error (RMSE) and mean absolute error (MAE) are selected to assess the performance of the proposed model. Results show that RMSE and MAE of the improved NeuMF model are 1.251 and 0.625 , respectively, which are better than collaborative filtering and traditional neural network factorization models. Moreover, the proposed model is used to recommend the activities of physical education (PE) for developing the intelligence of autistic children. This proves that the optimized model has better performance and can be used to recommend online courses for autistic users. This dynamic personalized curriculum recommendations model can help autistic children recover in a short time.
\end{abstract}

\section{Introduction}

Autism is a developmental disorder with symptoms that appear within the first three years of life. Its formal diagnostic name is an autism spectrum disorder. The word "spectrum" indicates that autism appears in different forms with varying levels of severity [1]. This means that each individual with autism experiences unique strengths, symptoms, and challenges. The most prominent deficits in autism are social communication, social interaction, and repetitive patterns of activities or behavior. Moreover, the cooccurrence of specific learning problems is also important in autism in terms of problems in acquiring academic skills markedly below age level and manifested in early school. Most individuals with autism have several learning problems. Therefore, it is hard to separate autism and learning difficulties in clinical diagnoses and manifestations [2].
At present, due to the influence of unhealthy family environments and inappropriate family education, the number of children with autism increases each year. This will cause an indelible psychological impact and affect the development of children [3]. Accordingly, there spring up many special education institutions to provide help to these children [4]. Several studies confirm the positive effects of various sources of physical education on the performance of children with autism, especially, their mental development, behavioral problems, motor and social skills, and muscle strength disruptive behavior. Studies have shown that improved health, including some psychosocial indicators in children with autism, is an outcome of applying specific types of physical education in the developed physical education programs [5]. Nowadays, there are several approaches to organizing PE for children with autism. Some authors are convinced that individual tasks are more significant in 
improving the psychophysical development and elaboration of social skills among children with autism [6]. With the development of the Internet age, such education is carried out online, which makes education convenient and timesaving. However, there also appear many problems. For example, teachers may not be able to observe the learning state of learners directly, and it is difficult to collect the information of learners after class so that they cannot bring appropriate curriculum that meets the needs of learners. Teachers have collected a large number of useful data in the process of network teaching, and how to use these data to reform education remains a hot topic in a long run. The emergence of artificial intelligence (AI) provides a good idea for constructing the recommendation system [7].

To optimize physical education for developing the intelligence of autistic children, several recommendation algorithms were integrated. Lampos et al. [8] proposed an AIbased approach for selecting effective teacher communication strategies in autism education and explored the potential of AI in autism education to help teachers' ineffective practice in developing social and educational outcomes for autistic children. A protocol was designed to detect such interactions and analyze the basic patterns in the collected observations. Moreover, machine learning techniques were employed to predict student response given the form of communication used by teachers under specific classroom conditions. Stevens et al. [9] determined the subgroups of autism by using machine learning algorithms such as Gaussian Mixture Models and Hierarchical Agglomerative Clustering. It was reported that machine learning can recognize the phenotypes of autism. Heinsfeld et al. [10] examined the application of deep learning algorithms for the identification of autistic children using the brain activation patterns. The prediction accuracy of this approach reached $70 \%$ which showed that machine learning methods are a very promising tool for the assessment of mental disorders. Kosmicki et al. [11] used machine learning algorithms to classify the people into autistic and nonautistic patients, and the results reached $98,81 \%$ of accuracy. The authors in [12] explored machine learning employing swarm intelligence to improve the accuracy and prediction of autism. Mirac et al. [13] observed some prediction factors after a 3-year clinical observation and psychiatric therapy of a group of autistic children and used machine learning methods to investigate the psychiatric, developmental, social, and demographic factors that affect the prognosis for children with autism. Crippa et al. [14] investigated the power of artificial intelligence to diagnose children with autism using kinematic analysis. The experiment involved a simple reach, grasp, and drop task with a ball, and the mean accuracy reached $84,9 \%$. The goal of the earliest recommendation algorithms was to bring personalized services to the people. Later, some scholars used the collaborative filtering (CF) method to build the recommendation system [15]. Based on the former, the item-based CF is introduced. With its continuous development, the recommendation system is classified, and it includes content, CF, and hybrid intelligent recommendation methods which are gradually applied in course teaching and provide many possibilities and personalized recommendation methods for online education
[16]. However, the recommendation accuracy decreases due to data missing, which results in data sparsity [17].

To reduce the data sparsity, this study integrated deep learning (DL) with $\mathrm{CF}$, and an improved Neu matrix factorization (improved NeuMF) is designed. The traditional matrix decomposition is used to find the linear relationship between users and items, and the multilayer neural network is used to explore the nonlinear relationship between users and items. The accuracy is also optimized to reduce the number of missing data. The time data are applied to improved NeuMF by using the K-means clustering algorithm (K-means) to maximize the optimization of online courses. After experiments, relevant evaluation indexes are selected to accomplish simulation tests on relevant datasets. The test results show that the performance of the model is good. The model designed based on improved NeuMF can provide appropriate activities of physical education (PE) for developing the intelligence of autistic children, which plays an important role and has great significance for the recovery of autistic students.

The rest of the manuscript is ordered as follows: Section 2 provides an overview of the materials and methods used and describes the proposed algorithm. In Section 3, the results are illustrated, and Section 4 is about the conclusion.

\section{Materials and Methods}

2.1. Problems in PE for Developing the Intelligence of Autistic Children. In real life, intelligent physical education (IPE) refers to special education carried out by formal educational institutions to make autistic students receive high-level education. They focus on the development and recovery of autistic children and provide skills training for them [18]. The training is usually conducted from the simple to the difficult and ultimately enables the children to have physical exercise suitable for their development. However, only when students with intellectual disabilities have the initiative to participate in PE, may cause unbalanced development. The problems in $\mathrm{PE}$ for developing the intelligence of autistic children are shown in Figure 1. The following section illustrates the different problems in $\mathrm{PE}$ for developing the intelligence of autistic children.

(i) Mismatch of the Teaching Content. At present, many teaching materials in special schools are adapted based on general education. Some teaching materials at this stage weaken the simplicity of high-tech projects, making the learning cycle disconnected. Although corresponding achievements have been made, the adapted textbooks still have a great gap with the needs of this kind of children [19].

(ii) Lack of Real-Life Application. Because the development state of autistic children restricts their normal development of bodies, high-tech activities are not attractive to them and may have a negative effect on their psychology. They are very interested in content that is closely related to real life. Unfortunately, the connection between the content of many courses and real life is weak [20]. 


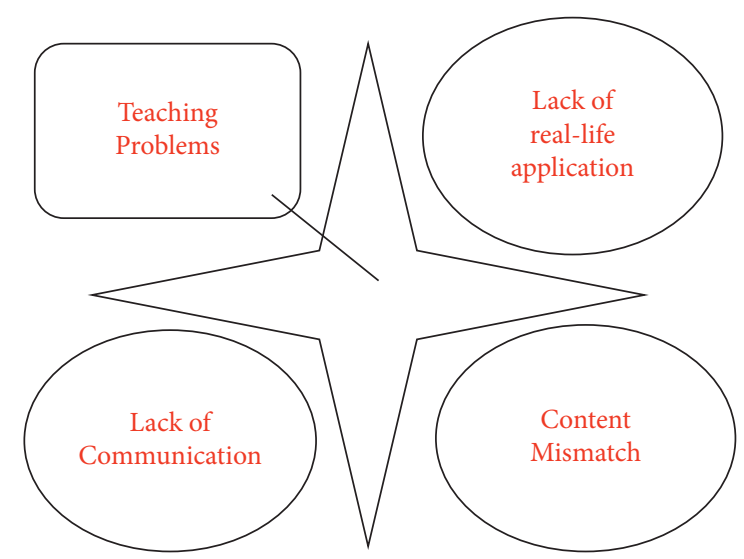

Figure 1: Problems in PE for developing the intelligence of autistic children.

(iii) Little Communication between Teachers and Students. The interaction between teachers and students is little in the teaching process in special schools. When teachers pay no attention to students, students' enthusiasm for the study will be greatly reduced, which will have a strong negative impact on the healthy development of autistic children [21].

With the continuous development of AI, the promotion of online education provides a breakthrough to solve the mismatch of the teaching content of PE and the children. However, there are still imperfections in the online curriculum recommendation algorithm, which needs to be improved.

2.2. Problems in Curriculum Recommendation. Before the curriculum recommendation algorithm can be proposed, there are still a few problems that need to be solved. These problems are depicted in Figure 2.

The problem of data sparsity exists because CF analyzes the users' previous information and uses it as the basis for relevant recommendations [22]. However, the ratings of related items may not be sufficient, and users may not know whether the ratings are accurate or not. After the user completes course learning, the rating may become an extra item and will not be complete, resulting in data sparsity. Data sparsity is one of the most important challenges in data in which each user only rates a small set of items. In real life, because there is no way to conduct face-to-face learning, there may appear the phenomenon that the rating may be performed casually leading to data sparsity. In the learning process, learning resources should be constantly updated, so that the accuracy of the recommendation algorithm can be confirmed. In addition, time is different from other auxiliary information such as users and course information. With the influx of other data, more time is needed [23].

Because of the above three problems, it is found that the neural network can extract high-level features and solve the problem of data sparsity in the nonlinear network of users

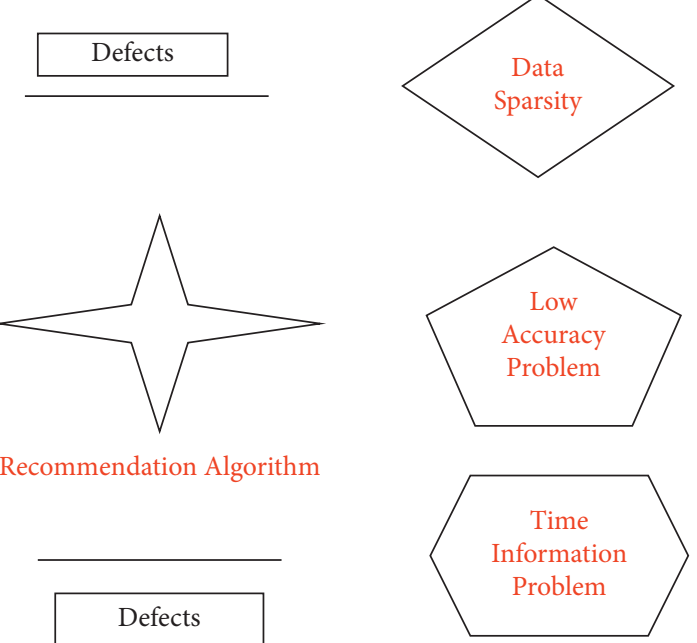

Figure 2: The problem of curriculum recommendation algorithm.

and items. The interference of previous courses on new recommendations can be reduced by adding the time into improved NeuMF, and the recommendation accuracy can be improved accordingly. In the face of the particularity of time, other data need to be optimized to upgrade and improve the model. Moreover, a comprehensive analysis of the shortcomings of the recommendation model is conducted.

2.3. Artificial Neural Networks. The integration of DL and online curriculum recommendation is of great practical significance. Online curriculum learning is adopted by many training institutions at present. As it is convenient and easy to access, students can learn a lot of knowledge without leaving home. However, the learning capabilities and states of the majority of students cannot be supervised due to the lack of face-to-face communication between teachers and students. And the gap between the teaching content and students' needs becomes greater and greater, and students will lose their interest in online courses. This problem can be solved using course recommendation systems. The integration of deep learning and the traditional recommendation algorithms can provide the individual needs of students [24].

As a model, the neural network can analyze complex problems according to their characteristics, and the relationship between the relevant nodes should continue to be adjusted. Neurons are the basic components of neural networks. Each neuron has multiple inputs, and the effect of input data on the whole neuron can be adjusted by weights. The higher the weight of an artificial neuron is, the stronger the input which is multiplied by it will be. Weights can also be negative, where the signal is inhibited by the negative weight. Based on the weights, the computation of the neuron will be different. By regulating the weights of an artificial neuron, the output for specific inputs can be obtained. When there are hundreds or thousands of neurons in an ANN, it would be quite difficult to find by hand all the necessary weights. However, we can find algorithms that can adjust the weights of the ANN to obtain the desired output from the 
network. This process of adjusting the weights is called learning or training. The continuous optimization of weights is to learn and train neural networks, and neurons can be expressed in image or text format. The neurons are mainly divided into input, output, and hidden units [25]. The neural network is composed of neurons, as shown in Figure 3.

The input layer of ANN receives external signals, the hidden layer is often between the input and output layer, and the output layer produces the output of the network. Using analysis of data processing, learning, and training, the appropriate solution is produced in the output layer. Weights are constantly adjusted according to the actual situation, which is called autonomous learning [26].

The neural network is determined by the structure of networks and is optimized using different hyperparameters. For example, there are activation functions, weights, and connection methods that are worthy of attention. Among the three, the activation function plays a vital role in the learning and training of ANN. In the relevant network, the nodes of each layer convert the input data into the output. The relevant activation function is needed to make a good conversation between the last output and the next input. It is not difficult to find that the so-called activation function is the functional relationship between the two. The neurons are input and mapped to the output by the activation function. An activation function in a neural network states how the weighted sum of the input is transformed into an output from a node in the network. When the activation function is used, its nonlinearity can change the input-output relationship of the network layer from linear to nonlinear to explore the deeper relationship. The selection of activation function has a significant impact on the performance of the neural network, and different activation functions may be used in different parts of the neural network model. The common activation functions are Sigmoid, Tanh, and ReLU, which are introduced in detail to pave the way for the later model design [27]. The range of Sigmoid is between 0 and 1 , and the results obtained by the input function are all between 0 and 1 . When the difference in item characteristics is not obvious, the effect is better, because the calculation of this function is complex and the gradient often disappears. The conversion equation between function $S$ and input $x$ is as follows:

$$
\begin{aligned}
S(x) & =\frac{1}{1+e^{-x}}, \\
S^{\prime} & =\frac{e^{-x}}{\left(1+e^{-x}\right)^{2}}=S(x)(1-S(x)) .
\end{aligned}
$$

Tanh solves the problems found in Sigmoid and is not symmetrical to the origin. It is a hyperbolic function and has the disadvantage of gradient saturation. The conversion between Tanh and input $x$ is calculated as follows:

$$
\operatorname{Tanh}(x)=\frac{e^{x}-e^{-x}}{e^{x}+e^{-x}} .
$$

Compared with Tanh and Sigmoid, ReLU has no saturation in its value, and the gradient does not decline [28]. On

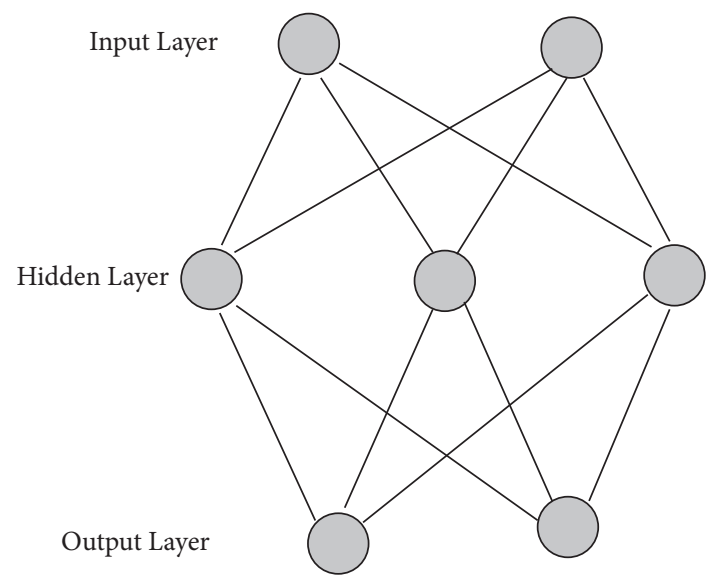

FIGURe 3: Artificial neural network.

calculating, the function is used to calculate the maximum, not the index, and the gradient has some other problems. The conversion equation between function ReLU and input $x$ is as follows:

$$
\begin{aligned}
f(x) & =\max (0, x), \\
\operatorname{ReLU}(x) & =\left\{\begin{array}{ll}
x, & \text { if } x>0 \\
0, & \text { if } x \leq 0
\end{array} .\right.
\end{aligned}
$$

2.4. CF Based on Neural Networks. Collaborative filtering filters information by using the interactions and data collected by the system from other users. The CF is based on the idea that users who agreed in their evaluation of certain items are expected to agree again in the future. Most collaborative filtering approaches use similarity index-based techniques. Several users are selected based on their similarity to the active user, and inference for the active user is made by calculating a weighted average of the ratings of the selected users. Its working principle is to judge the users' preferences by analyzing their previous data. According to these preferences, users are classified and grouped. Users with similar preferences are recommended similar courses [28]. The recommendation based on CF is optimized by researchers, expecting to improve its performance. Previously, in CF many matrix factorization (MF) methods were used to construct hidden feature correlations between users and items. However, a simple inner product cannot estimate the complex correlation, and the accuracy of recommendation is affected [29]. Therefore, an improved neural network matrix factorization (NeuMF) is proposed, where the neural network replaces the inner product in the previous matrix, which can help to reveal the linear and nonlinear relations between users and items. The framework of improved NeuMF is shown in Figure 4.

Figure 4 shows that improved NeuMF has four levels, namely, the input layer, the embedded layer, the NeuMF layer, and the output layer. The input layer is to input users' information and items. It also transforms each user and item into a vector [30]. If there are $n$ users, it will be converted 


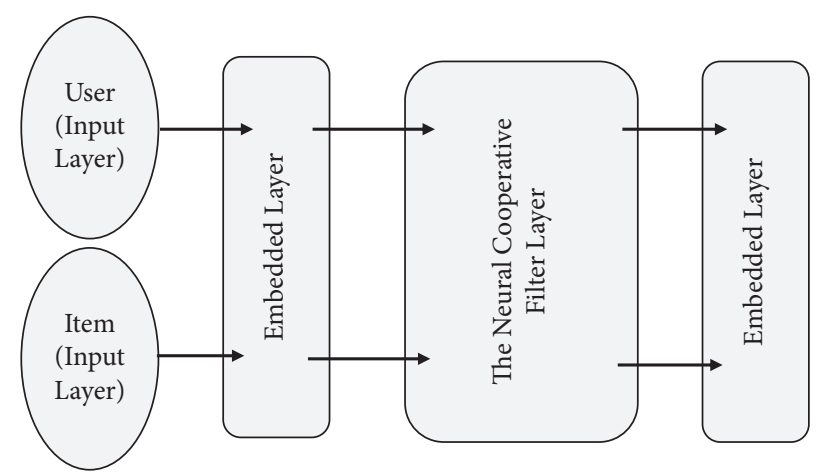

Figure 4: The framework of neural collaborative filtering.

into a vector of $1 \times n$ users, which will be converted into sparse vectors later. After the input arrives at the embedding layer, the input vector is multiplied by the embedding matrix. If there are $n$ users and the embedding dimension is $m$, the size of the embedding matrix is $m \times n$. The line refers to the embedding vector of the relevant users. After the users' and item embedding matrices are obtained by the NeuMF layer, the output layer outputs the final results.

2.4.1. Generalized Matrix Factorization Model. Matrix factorization deposes a matrix into a product of 1 or $n$ matrices to overcome the shortcomings of data sparsity in the traditional cooperative filtering algorithm [31]. The left side of the neural collaborative filtering model is the generalized matrix factorization model (GMF), which uses the product of vectors to perform calculations, and the final result is also a vector. The equation of GMF is as follows:

$$
\begin{aligned}
p_{u} & =\left[p^{1}, \ldots \ldots, p^{k}\right], \\
q_{i} & =\left[q^{1}, \ldots \ldots, q^{k}\right], \\
\varphi\left(p_{u}, q_{i}\right) & =p_{u} \cdot q_{i}=\left[p^{q} q^{q}, p^{2} q^{2}, \ldots \ldots, p^{k} q^{k}\right], \\
y_{\text {ui }} & =\alpha_{\text {out }}\left(h\left(p_{u} \cdot q_{i}\right)\right),
\end{aligned}
$$

where $p_{u}$ and $q_{i}$ are the potential feature vectors of users and items input into the embedding layer. The correlation between users and items is obtained by the inner product, and then the final prediction results are output through the output layer. Likewise, a, $u$, and $t$ are the activation functions of Sigmoid, and $\mathrm{h}()$ is the output layer [32].

2.4.2. ANN with a Forward Structure. Multilayer perceptron (MLP) is an ANN with a forward structure. It consists of multiple layers, including the input layer, hidden layer, and output layer. All these layers are fully connected, and data are transmitted forward between them. After each output is calculated by the algorithm, the backpropagation (BP) algorithm is used to find the optimal parameters. If the data entered in the input layer is $(a, b)$ and the weights of the hidden and output layer are w1 and $\mathrm{w} 2$, the equations of the functions $\mathrm{a}, u$, and $t$ obtained from the hidden layer are

$$
\begin{aligned}
\alpha_{h} & =f_{1}\left(w_{1} \cdot \alpha\right), \\
\alpha_{\text {out }} & =f_{2}\left(w_{2} \cdot \alpha_{h}\right) .
\end{aligned}
$$

Improved NeuMF is next to the MLP framework. The network structure of MLP can find the deeper correlation between users and items, as shown in Figure 5.

Figure 5 shows that the feature vectors Pu and Qi of users and items are used as input into MLP to find the final score, which is different from GMF in dealing with the embedding vectors of users and items in the embedding layer.

2.5. Construction of Improved NeuMF with Time Fusion. In practice, the time has a relatively large effect on online curriculum resources. Generally, it is believed that the user's preference is fixed and does not change. But in the real teaching process, the user's preference changes with time. Therefore, in addition to DL, relevant auxiliary information can make the recommendation effect better [32]. Time is fused into the recommendation algorithm to better explore the dynamic preferences of users. As compared to the existing recommendation models, the greatest factor influencing the recommendation effect is time. With the continuous improvement of learning, the curriculum resources should be renewed because the learning direction of each user changes in different stages. In this study, the popularity of the courses is also presented along with time. Moreover, the time is classified by $\mathrm{K}$-means and it is fused into MLP and GMF as a time feature vector, providing users with a more accurate dynamic recommendation.

$\mathrm{K}$-means is an algorithm for partitioning clustering. In K-means, several data on the dataset are randomly selected as the initial values. Next, they are compared with the obtained values, and the comparison is to calculate the distance between the central value and each datum. Then, the calculation results reveal the correlation between the data and the center, and each datum is assigned to the nearest cluster center. Finally, the cluster center and each datum next to it form a cluster. If the cluster center is changed, the process is repeated; otherwise, the process is terminated. Through this method, the data can be divided into several categories. Distance $y$ is expressed as 


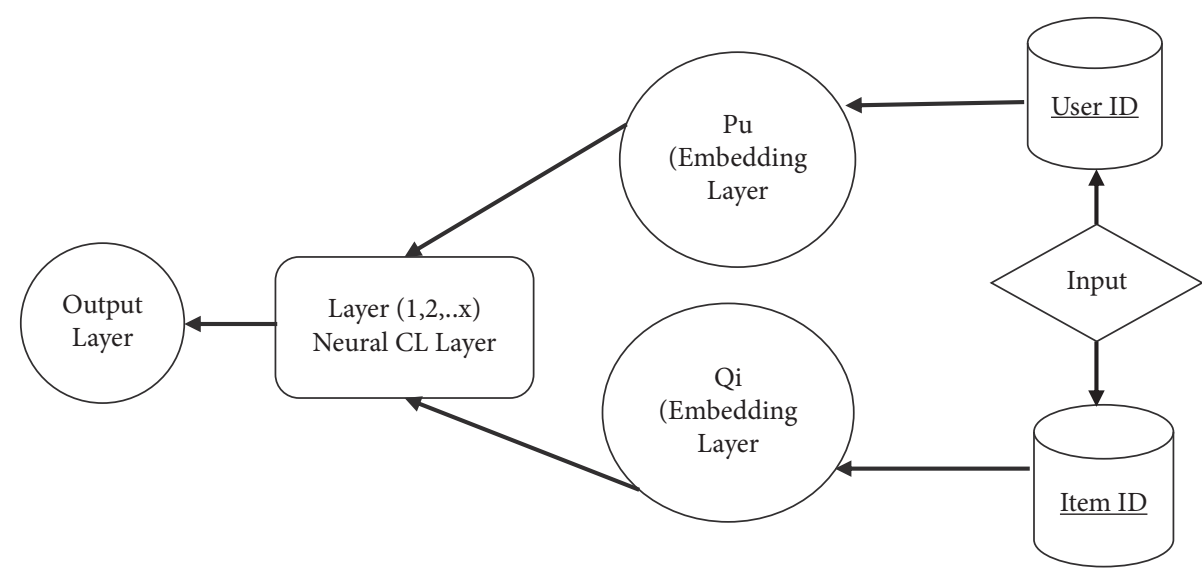

FIgURe 5: MLP framework.

$$
y=\min \sum_{i=1}^{n} \min _{j=1,2, . . k} x_{i}-\mu^{2},
$$

where $\mathrm{xi}$ is the data on the dataset, $\mu$ is the clustering center value, and $K$ is the number of initial clusters. $\mathrm{K}$-means finds the correlation between data and classifies them in the cluttered data. Time can be infinitely large, which proves that time can be unilaterally used as auxiliary information in the model. In this case, the complexity of the model greatly increases. Therefore, K-means is used to solve the problem and map time into an interval. In this way, improved NeuMF is designed, and its framework is shown in Figure 6.
In the learning and training of the proposed model, the convergence speed of the training may be affected and slowed down with an increase in the number of network layers. With this regard, batch standardization is performed according to relevant standards, and the distribution of the input values of each layer is adjusted to speed up the training speed. The batch standardization layer is added into MLP to improve the training speed and alleviate overfitting. After linear learning and nonlinear learning, the obtained feature vectors are connected, and the Sigmoid activation function is used to output the results. The improved NeuMF is computed as

$$
\begin{aligned}
X^{\mathrm{GMF}} & =p_{u}^{\mathrm{GMF}} \cdot q_{i}^{\mathrm{GMF}}+p_{i}^{\mathrm{GMF}} \cdot t_{u i}^{\mathrm{GMF}}, \\
X^{\mathrm{MLP}} & =\alpha_{L}\left(W_{L}^{T}\left(\alpha y_{u i}=\sigma\left(h^{T}\left[\begin{array}{c}
X^{\mathrm{GMF}} \\
X^{\mathrm{MLP}}
\end{array}\right]\right)\right)_{L-1}\left(\ldots \alpha_{2}\left(W_{2}^{T}\left[\begin{array}{c}
p_{u}^{M} \\
\left.\left.\left.q_{i}^{M}\right]+b_{2}\right) \ldots\right] \\
t_{u i}^{M}
\end{array}\right]\right)+b_{L}\right),\right. \\
y_{u i} & =\sigma\left(h^{T}\left[\begin{array}{l}
X^{\mathrm{GMF}} \\
\left.\left.X^{\mathrm{MLP}}\right]\right)
\end{array}\right)\right. \\
L & =\frac{1}{n} \sum_{i=1}^{n}\left|y_{u i}-y_{\text {real }}\right|^{2}, \\
L & =\sum_{(u, i) \in D \cup D^{-}}\left[y_{u i} \log y_{\text {real }}-\left(1-y_{u i}\right) \log \left(1-y_{\text {real }}\right)\right],
\end{aligned}
$$




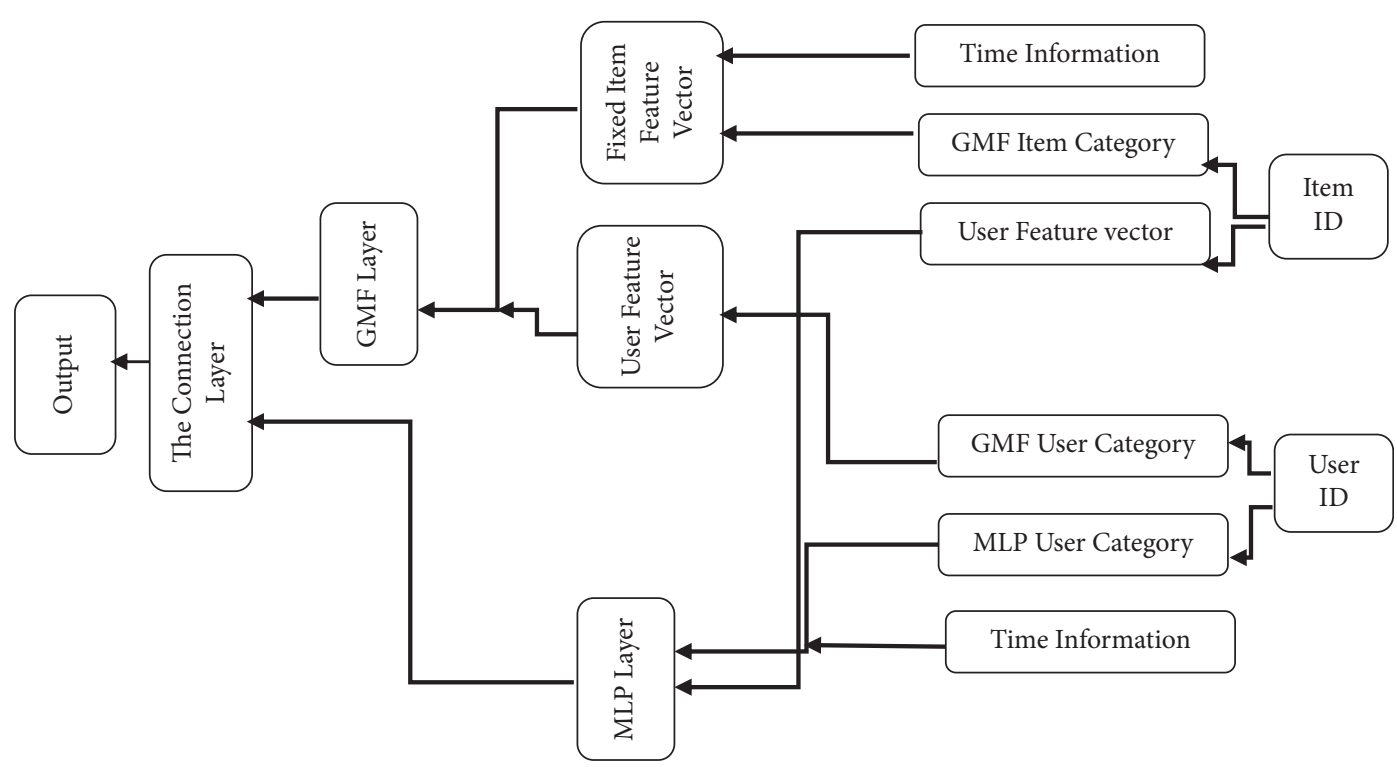

FIgURE 6: Improved NeuMF.

where $p_{u}^{M}$ and $p_{u}^{G M F}$ are the inputs of the user in MLP and GMF, $t_{u i}^{G M F}$ is the time spent by the user on item $i$ in GMF, and the viewing time classified in MLP is input as one-dimensional vectors.

\section{Results}

There are many online course platforms, such as MOOC and Netease Cloud Classroom. In this study, the Scrapy in python is used to crawl the data of MOOC, and a total of 289333 data are collected, from which more than 13 user records are randomly selected and 6689 users and 478 courses on the dataset are obtained. The root means square error (RMSE) and means absolute error (MAE) of CF, Neu matrix factorization (NeuMF), and improved NeuMF are compared, and the results are analyzed.

3.1. Performance Comparison. We compared different methods for course recommendation. Among the 6689 users and 544 courses, the course that users have learned is selected as the test set and the remaining courses were used as the training set. A total of 100 courses that users learned were added to the test set. To compare the performance of NeuMF, improved NeuMF, and CF, we computed RMSE and MAE of the data for all three algorithms. The comparative results are shown in Table 1.

It can be seen that RMSE and MAE of improved NeuMF are 1.251 and 0.625 , respectively. Similarly, the RMSE and MAE for NeuMF are 1.372 and 0.825 , respectively. The collaborative filtering reported the highest values of 3.362 for RMSE and 2.953 for MAE. It is evident that as compared to the other two algorithms, the error produced by the proposed improved NeuMF is significantly smaller and its accuracy is higher. The values of each of these algorithms are put into the dot-line diagram to highlight the gap between the obtained results, as shown in Figure 7.
TABLE 1: Comparison results of RMSE and MAE.

\begin{tabular}{lccc}
\hline & NeuMF & Collaborative filtering & Improved NeuMF \\
\hline RMSE & 1.372 & 3.362 & 1.251 \\
MAE & 0.825 & 2.953 & 0.625 \\
\hline
\end{tabular}

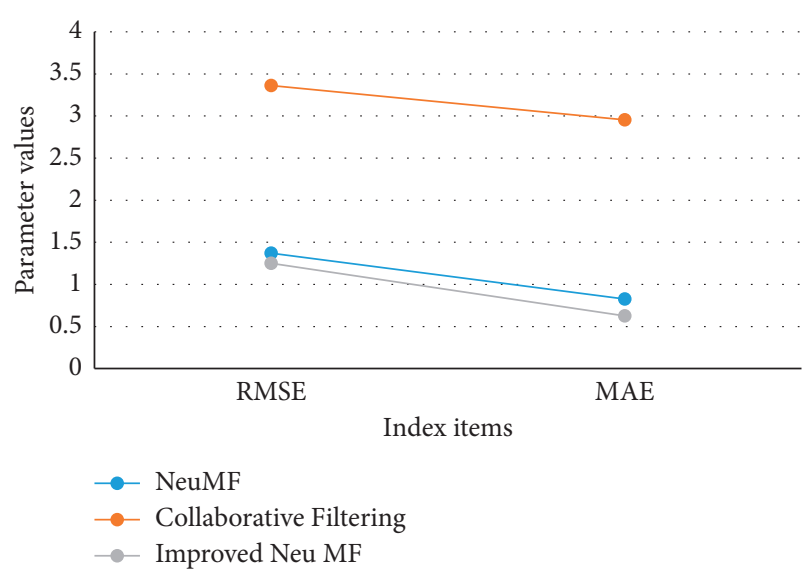

FIgure 7: Comparison of the gap between MAE and RMSE.

The comparison shows that RMSE and MAE of improved NeuMF are about 1\% lower than those of NeuMF and CF, and its accuracy is higher.

3.2. Comparison of Accuracy and Sequential Experiments of Different Algorithms. Various evaluation metrics are used for evaluating the effectiveness of a recommendation system. To evaluate the efficiency of the different recommendation systems, we employed Hit Ration (HR) and Normalized Discounted Cumulative Gain (NDCG). HR can reflect the accuracy of recommendation and is simply the fraction of users for which the correct answer is included in the recommendation list and NDCG can present the order of 
TABLE 2: Experimental results of HDCG and HR.

\begin{tabular}{lcc}
\hline Algorithms & Normalized discounted cumulative gain & Hits ratio \\
\hline NeuMF & 0.32 & 0.37 \\
Collaborative filtering & 0.06 & 0.11 \\
Improved NeuMF & 0.42 & 0.51 \\
\hline
\end{tabular}

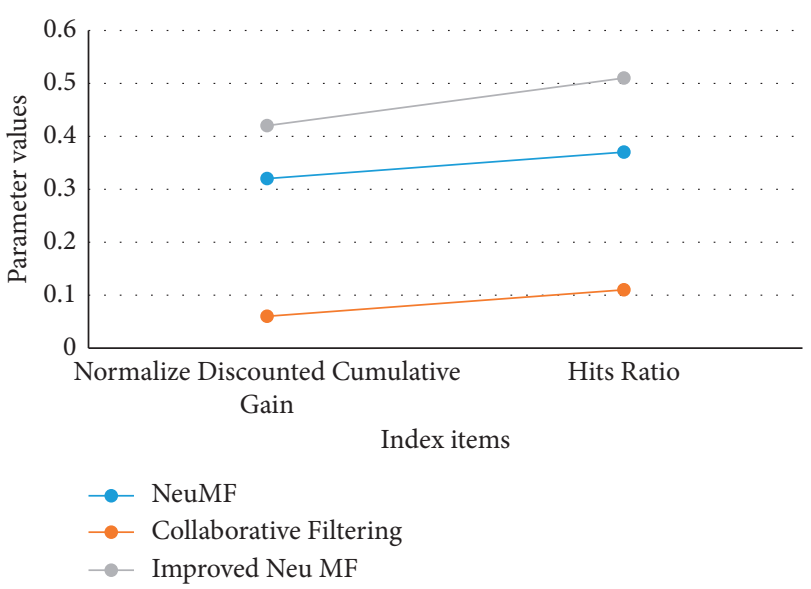

FIgURE 8: Comparison of the gaps of HDCG and HR.

recommended items. The three algorithms are compared in terms of HR and NDCG, and the results are shown in Table 2 .

Table 2 shows that NDCG and HR of improved NeuMF are 0.42 and 0.51 , respectively, whereas those of NeuMF and collaborative filtering are $0.32,0.37$ and $0.06,0.11$, respectively. The addition of time can help to find more suitable courses for users. The gaps of the above algorithms are displayed in Figure 8.

In the figure, NDCG and HR of improved NeuMF are higher than others. It can be concluded that NDCG and HR of improved NeuMF are about 0.4 higher than CF. Therefore, the performance of the designed algorithm is more excellent when time is added.

\section{Conclusion}

There are more and more autistic children due to the influence of unhealthy family environments and inappropriate family education. This study is conducted to help them recover in $\mathrm{PE}$ by recommending more suitable online courses for these children. In this process, the advantages of DL and CF are integrated to design an improved NeuMF. The matrix decomposition is used for the linear relationship between users and items, and MLP is employed for capturing the nonlinear relationship between them. Since with the progress of teaching, curriculum resources are changed accordingly, time is added to CF by K-means to maximize the optimization of online curriculum resources. Relevant evaluation indexes are selected to operate simulation tests on relevant datasets. The test results show that the model proposed has the optimal performance compared with others. Based on DL, the model designed can provide better curriculum recommendations for autistic children, which plays an important role in their recovery. In this study, only individual learning platforms are selected in the data selection, which may have a certain impact on the experimental results. In the subsequent research, the size of sample data will be expanded to make the experimental results more convincing.

\section{Data Availability}

The experimental data used to support the findings of this study are available from the corresponding author upon request.

\section{Conflicts of Interest}

The authors declare that they have no conflicts of interest regarding this work.

\section{Acknowledgments}

This work was supported by the Humanities and Social Sciences General Project of Anhui Universities in 2019 "Study on the development PATH of adaptive P.E. curriculum in Anhui Universities from the perspective of inclusive education" (Project no.2019SK22) and by Teaching Quality and Teaching Reform Project of Huaibei Normal University in 2020 "Research on curriculum and Teaching Reform of 'Education for Autistic Children' from the perspective of Embodied Cognition" (Project no.2020xjxyj036).

\section{References}

[1] C. Ou, D. A. Joyner, and A. K. Goel, "Designing and developing video lessons for online learning: a seven-principle model," Online Learning, vol. 23, no. 2, pp. 82-104, 2019.

[2] P.-Y. Chen, X. Peng, and S. Yu, "NeuroSim: a circuit-level macro model for benchmarking neuro-inspired architectures in online learning," IEEE Transactions on Computer-Aided Design of Integrated Circuits and Systems, vol. 37, no. 12, pp. 3067-3080, 2018.

[3] P. Timonen and H. Ruokamo, "Designing a preliminary model of coaching pedagogy for synchronous collaborative online learning," Journal of Pacific Rim Psychology, vol. 15, Article ID 1834490921991430, 2021.

[4] I. Esteban-Millat, F. J. Martínez-López, M. Pujol-Jover, J. C. Gázquez-Abad, and A. Alegret, "An extension of the technology acceptance model for online learning environments," Interactive Learning Environments, vol. 26, no. 7, pp. 895-910, 2018.

[5] H.-C. K. Hsu, C. V. Wang, and C. Levesque-Bristol, "Reexamining the impact of self-determination theory on learning outcomes in the online learning environment," Education and Information Technologies, vol. 24, no. 3, pp. 2159-2174, 2019.

[6] V. Singh and A. Thurman, "How many ways can we define online learning? a systematic literature review of definitions of 
online learning (1988-2018)," American Journal of Distance Education, vol. 33, no. 4, pp. 289-306, 2019.

[7] R. Wong, "When no one can go to school: does online learning meet students' basic learning needs?" Interactive Learning Environments, vol. 72, pp. 1-17, 2020.

[8] V. Lampos, J. Mintz, and X. Qu, "An artificial intelligence approach for selecting effective teacher communication strategies in autism education," Npj Science of Learning, vol. 6, no. 1, p. 25, 2021.

[9] E. Stevens, D. R. Dixon, M. N. Novack, D. Granpeesheh, T. Smith, and E. Linstead, "Identification and analysis of behavioral phenotypes in autism spectrum disorder via unsupervised machine learning," International Journal of Medical Informatics, vol. 129, pp. 29-36, 2019, Available: https://www.sciencedirect.com/science/article/\%20pii/ S138650561830.

[10] A. S. Heinsfeld, A. R. Franco, C. Craddock, A. Buchweitz, and F. Meneguzzia, "Identification of autism spectrum disorder using deep learning and the ABIDE dataset," Clinic, vol. 17, pp. 16-23, 2017, Available: https://www.meta.org/papers/\% 20identification-of-autism-spectrum-disorder-usi.

[11] JA. Kosmicki, V. Sochat, M. Duda, and DP. Wall, "Searching for a minimal set of behaviors for autism detection feature selection-based machine learning," Translational Psychiatry, vol. 5, no. 514, pp. 1-7, 2015.

[12] R. Vaishali and R. Sasikala, "A machine learning-based approach to classify autism with optimum behavior sets," International Journal of Engineering and Technology, vol. 4, no. 4, pp. 4216-4219, 2018.

[13] B. U. Mirac, K. Karabekiroglu, B. Sahin et al., "Use of machine learning methods in prediction of short-term outcome in autism spectrum disorders," Psychiatry and Clinical Psychopharmacology, vol. 29, Article ID 1545334, 2018.

[14] A. Crippa, C. Salvatore, P. Perego et al., "Use of machine learning to identify children with autism and their motor abnormalities," Journal of Autism and Developmental Disorders, vol. 45, no. 7, pp. 2146-2156, 2015.

[15] I. AlQerm and B. Shihada, "Energy-efficient power allocation in multitier 5G networks using enhanced online learning," IEEE Transactions on Vehicular Technology, vol. 66, no. 12, pp. 11086-11097, 2017.

[16] A. H. Aldholay, O. Isaac, Z. Abdullah, A. Ibrahim, and N. Mohammed, "The role of compatibility as a moderating variable in the information system success model: the context of online learning usage," International Journal of Management and Human Science (IJMHS), vol. 2, no. 1, pp. 9-15, 2018.

[17] M. M. Idrees, L. L. Minku, F. Stahl, and A. Badii, "A heterogeneous online learning ensemble for non-stationary environments," Knowledge-Based Systems, vol. 188, Article ID 104983, 2020.

[18] M. Kersting, E. K. Henriksen, M. V. Bøe et al., "General relativity in upper secondary school: design and evaluation of an online learning environment using the model of educational reconstruction," Physical Review Physics Education Research, vol. 14, no. 1, Article ID 010130, 2018.

[19] K. K. Bhagat, L. Y. Wu, and C. Y. Chang, "The impact of personality on students' perceptions towards online learning," Australasian Journal of Educational Technology, vol. 35, no. 4, 2019.

[20] J. B. Wandler and W. J. Imbriale, "Promoting undergraduate student self-regulation in online learning environments," Online Learning, vol. 21, no. 2, p. n2, 2017.
[21] Z. Cui, X. Xu, F. Xue et al., "Personalized recommendation system based on collaborative filtering for IoT scenarios," IEEE Transactions on Services Computing, vol. 13, no. 4, pp. 685-695, 2020.

[22] H. Gao, Y. Xu, Y. Yin, and W. Zhang, "Context-aware QoS prediction with neural collaborative filtering for Internet-ofThings services," IEEE Internet of Things Journal, vol. 7, no. 5, pp. 4532-4542, 2019.

[23] F. Xue, X. He, X. Wang, J. Xu, K. Liu, and R. Hong, "Deep item-based collaborative filtering for Top-N recommendation," ACM Transactions on Information Systems, vol. 37, no. 3, pp. 1-25, 2019.

[24] D. Kluver, M. D. Ekstrand, and J. A. Konstan, "Rating-based collaborative filtering: algorithms and evaluation," Social Information Access, vol. 10100, pp. 344-390, 2018.

[25] N. Nassar, A. Jafar, and Y. Rahhal, "A novel deep multicriteria collaborative filtering model for recommendation system," Knowledge-Based Systems, vol. 187, Article ID 104811, 2020.

[26] M. Nilashi, O. Ibrahim, and K. Bagherifard, "A recommender system based on collaborative filtering using ontology and dimensionality reduction techniques," Expert Systems with Applications, vol. 92, pp. 507-520, 2018.

[27] R. Yuan and P. Stapleton, "Student teachers' perceptions of critical thinking and its teaching," ELT Journal, vol. 74, no. 1, pp. $40-48,2020$.

[28] C.-H. Chen and Y.-C. Yang, "Revisiting the effects of projectbased learning on students' academic achievement: a metaanalysis investigating moderators," Educational Research Review, vol. 26, pp. 71-81, 2019.

[29] A. J. Guimaraes, V. J. S. Araujo, V. S. Araujo, L. O. Batista, and P. V. C. Souza, "A hybrid model based on fuzzy rules to act on the diagnosed of autism in adults," in Proceedings of the IFIP International Federation for Information Processing 2019, pp. 401-412, Portugal, May 2019.

[30] M. Duda, J. Daniels, and D. P. Wall, "Clinical evaluation of a novel and mobile autism risk assessment," Journal of Autism and Developmental Disorders, vol. 46, no. 6, pp. 1953-1961, 2016.

[31] S. M. Kanne, L. A. Carpenter, and Z. Warren, "Screening in toddlers and preschoolers at risk for autism spectrum disorder: evaluating a novel mobile-health screening tool," Autism Research, vol. 11, no. 7, pp. 1038-1049, 2018.

[32] K. A. A. Mamun, S. Bardhan, A. Ullah et al., "Smart autism - a mobile, interactive and integrated framework for screening and confirmation of autism," Proceedings of the Annual International Conference of the IEEE Engineering in Medicine and Biology Society. IEEE Engineering in Medicine and Biology Society, pp. 5989-5992, 2016. 